KCNMB1 transcriptional regulation has been performed $(9,10)$, the genomic structure of the promoter appears to share elements with other smooth muscle specific genes, which contain important regulatory elements in a large first intron $(11,12)$. Exposure of vascular myocytes to pressors such as angiotensin, used here to produce hypertension, results in transcriptional activation of numerous genes, through angiotensin II type 1 receptor (AT1) activation (13). Whether these target genes include pore-forming or regulatory ion channel subunits is yet to be determined.

The $\beta$ test described here by Amberg et al. (7) further highlights the unique, localized $\mathrm{Ca}^{2+}$ signaling mechanism in vascular myocytes and the role of the $\beta$ subunit in tuning the $\mathrm{Ca}^{2+}$ sensitivity of the BK channel complex. Functional changes in coupling between the SR and the sarcolemma, resulting from the altered expression of BK $\beta$ subunits, may be a key mechanism underlying variations in vascular tone in vivo. This idea, and the mechanism by which angiotensin and perhaps other pressors regulate $\beta$ subunit expression, will undoubtedly be the subject of considerable future investigation.

1. Nelson, M.T., et al. 1995. Relaxation of arterial smooth muscle by calcium sparks. Science. 270:633-637.

2. Orio, P., Rojas, P., Ferreira, G., and Latorre, R. 2002. New disguises for an old channel: MaxiK channel beta-subunits. News Physiol. Sci. 17:156-161.

3. Knaus, H.G., et al. 1994. Primary sequence and immunological characterization of $\beta$-subunit of high conductance $\mathrm{Ca} 2+$-activated $\mathrm{K}+$ channel from smooth muscle. J. Biol. Chem. 269:17274-17278.

4. Valverde, M.A., et al. 1999. Acute activation of Maxi$\mathrm{K}$ channels (hSlo) by estradiol binding to the beta subunit. Science. 285:1929-1931.

5. Brenner, R., et al. 2000. Vasoregulation by the beta1 subunit of the calcium-activated potassium channel. Nature. 407:870-876.

6. Pluger, S., et al. 2000. Mice with disrupted BK channel beta1 subunit gene feature abnormal $\mathrm{Ca}(2+)$
spark/STOC coupling and elevated blood pressure. Circ. Res. 87:E53-E60.

7. Amberg, G.C., Bonev, A.D., Rossow, C.F., Nelson, M.T., and Santana, L.F. 2003. Modulation of the molecular composition of large conductance, $\mathrm{Ca}^{2+}$ activated $\mathrm{K}^{+}$channels in vascular smooth muscle during hypertension J. Clin. Invest. 112:717-724. doi:10.1172/JCI200318684.

8. Caulfield, M., et al. 2003. Genome-wide mapping of human loci for essential hypertension. Lancet. 361:2118-2123.

9. Dhulipala, P.D., and Kotlikoff, M.I. 1999. Cloning and characterization of the promoters of the maxiK channel alpha and beta subunits. Biochim. Biophys. Acta. 1444:254-262.

10. Jiang, Z., Wallner, M., Meera, P., and Toro, L. 1999. Human and rodent MaxiK channel beta-subunit genes: cloning and characterization. Genomics. 55:57-67.

11. Mack, C.P., and Owens, G.K. 1999. Regulation of smooth muscle alpha-actin expression in vivo is dependent on CArG elements within the $5^{\prime}$ and first intron promoter regions. Circ. Res. 84:852-861.

12. Madsen, C.S., et al. 1998. Smooth muscle-specific expression of the smooth muscle myosin heavy chain gene in transgenic mice requires 5 '-flanking and first intronic DNA sequence. Circ. Res. 82:908-917.

13. Kim, S., and Iwao, H. 2000. Molecular and cellular mechanisms of angiotensin II-mediated cardiovascular and renal diseases. Pharmacol. Rev. 52:11-34.

\title{
The host response to anthrax lethal toxin: unexpected observations
}

\section{Alice S. Prince \\ Department of Pediatrics, Columbia University College of Physicians and Surgeons, New York, New York, USA}

\begin{abstract}
Bacillus anthracis, the causative agent of anthrax, is believed to induce disease and death in humans in an endotoxic shock-like manner. A comprehensive study (see the related article beginning on page 670) of the effects of anthrax toxin in mice demonstrates that toxin-induced death is mediated not by cytokine release, as previously thought, but by hypoxia-induced liver failure. The study strongly suggests that the therapies developed for treatment of cytokine-mediated septic shock will not be appropriate for the treatment of anthrax.
\end{abstract}

\section{J. Clin. Invest. 112:656-658 (2003). doi:10.1172/JCI200319581.}

Anthrax is an ancient disease now believed to be the cause of two of the plagues of Egypt described in the Old

\footnotetext{
Address correspondence to: Alice S. Prince, Department of Pediatrics, Columbia University College of Physicians and Surgeons, 650 West 168th Street, Black Building 418, New York, New York 10032, USA. Phone: (212) 305-4193; Fax: (212) 305-2284;

E-mail: asp7@columbia.edu.

Conflict of interest: The author has declared that no conflict of interest exists.

Nonstandard abbreviations used: anthrax toxin receptor (ATR).
}

Testament. It was further characterized by the Romans in the writings of Virgil in $28-27$ B.C. (1), and studied in a systematic fashion by the preeminent 19th-century scientists Robert Koch and Louis Pasteur (2). The first anthrax vaccine was produced by Pasteur in 1881 for use in cattle and was the predecessor to the more immunogenic attenuated strain of Bacillus anthracis developed in the 1930s by Sterne (3). As early as the 19th century there were insights into the immunodominant and protective antigens expressed by the organism. The essential virulence factors of $B$. anthracis are known and well characterized (4); the bacterial adhesin has been identified, its binding domain mapped, and its crystal structure determined at a resolution of $2 \AA$ (5). The anthrax toxin receptor (ATR), an integral membrane protein, has been identified (6). The genomic DNA of $B$. anthracis has been sequenced; critical domains of the major virulence factors are mapped, and much of the molecular biology of the major virulence determinants of anthrax is well established, at least in vitro. Yet, despite the long history of $B$. anthracis as a human and animal pathogen and its notoriety as an agent of biological warfare, exactly how anthrax kills the host is unclear. In an exhaustive pathological study presented in this issue of the JCI, observations from the laboratory of Stephen Leppla and colleagues provide major new insights into the pathogenesis of anthrax (7).

\section{Pathogenesis of anthrax: plasmid-mediated expression of essential virulence factors}

Through the work of many investigators it has been well established that virulent $B$. anthracis expresses two plasmids, pXO1 and pXO2. The plasmid pXO1 expresses the anthrax toxins 


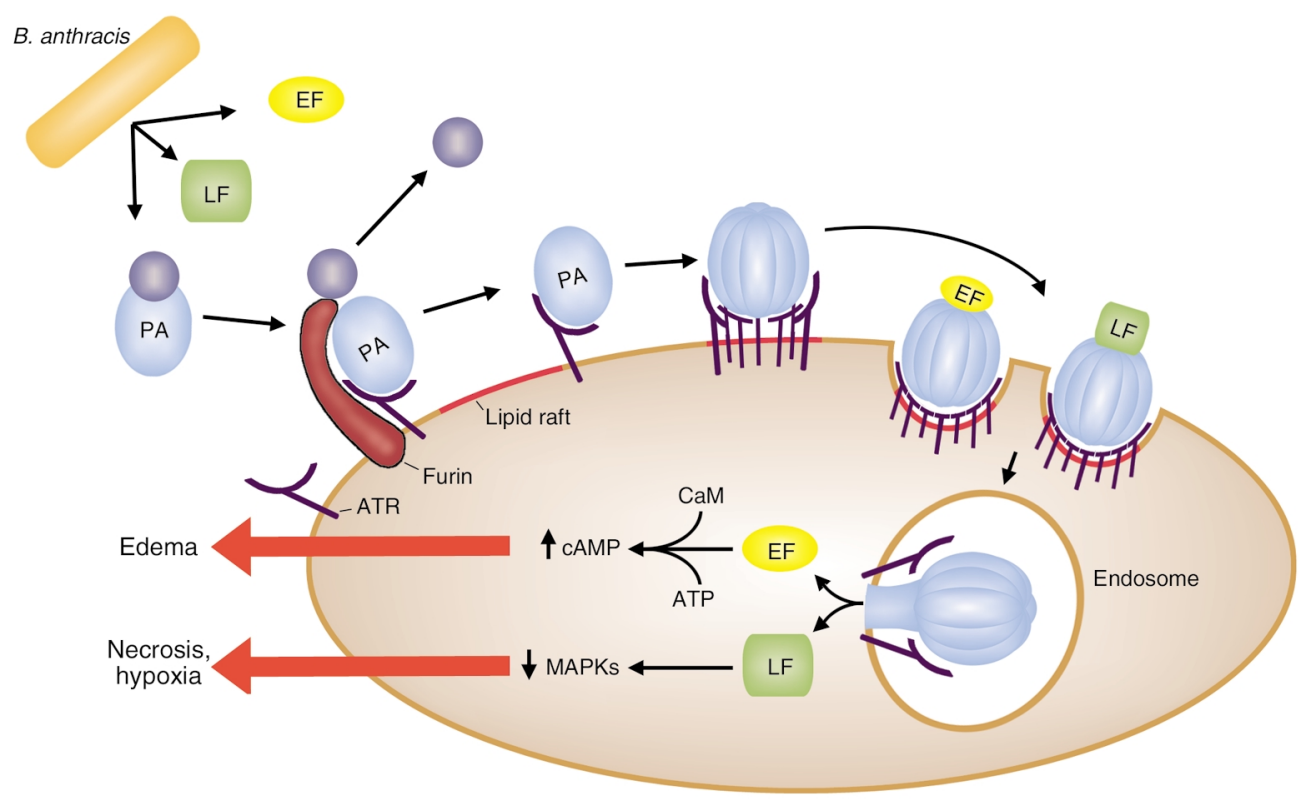

\section{Figure 1}

How anthrax toxins cause pathology. Fully virulent $B$. anthracis produce an antiphagocytic capsule as well as toxins. The protective antigen (PA) of the anthrax toxin binds to the ATR on the host cell surface. The $83-\mathrm{kDa}$ form of PA is cleaved by the cell surface protease furin and produces a 63-kDa monomer. Heptamerization of PA induces clustering of the ATRs, association of the complex with lipid rafts, and exposure of binding domains to the edema factor (EF) or the lethal factor (LF). The heptamer, and bound EF or LF, are then endocytosed. EF, an adenylate cyclase, and LF, a $\mathrm{Zn}^{2+}$ metalloprotease, translocate to the cytosol through a pore created in the membrane and act on host cytosolic targets to induce edema, necrosis, and hypoxia. Modified with permission from Annual Reviews (4). CaM, calmodulin.

lethal factor and edema factor, as well as protective antigen, which functions as a ligand. The genes necessary for capsule formation are encoded on pXO2. Both plasmids are required for virulence. The spontaneous loss of $\mathrm{pXO} 2$ results in the failure to produce the polyglutamate capsule and was the basis for the early attenuated vaccine strain described by Sterne. While the expression of a polypeptide capsule is somewhat unusual, its role in pathogenesis - to inhibit phagocytosis - is a function similar to that of many other bacterial capsules. Less straightforward are the effects of the toxins produced by pXO1, the lethal factor and the edema factor.

The toxins of $B$. anthracis can be considered "classic" AB toxins, in which the protective antigen functions as the binding component, providing entry for either of the two toxins into the host cell (8). The protective antigen binds the edema factor, a calmodulindependent adenylate cyclase (9) with homology to a similar enzyme in Bordetella pertussis, and the lethal factor, a zinc metalloproteinase that cleaves MAPKs (10). Once the protective antigen is bound to the host cell via the
ATR, it is processed by a furin-family protease into a $63-\mathrm{kDa}$ form that oligomerizes into a heptamer (11). This cleavage is necessary to expose the binding sites for the lethal factor and/or the edema factor. It appears that each monomer of the protective antigen binds either the edema factor or the lethal factor, thus providing access for seven molecules of toxin to enter the host cell. Recent studies demonstrate that the cleaved protective antigen and its receptor are clustered into lipid rafts and then internalized via the classic clathrin-dependent endocytic pathway (12), thus providing the toxin molecules access to their targets, including the MAPKs and ATP (Figure 1). In vitro, the activity of these toxins has been well established. The edema factor binds both calmodulin and ATP, which is converted to cAMP, thereby activating fluid secretion and causing edema. The production of lethal factor, the zinc metalloproteinase, has been shown to cause cell lysis usually within 1-2 hours.

\section{Clinical manifestations of anthrax}

The clinical manifestations of anthrax are also well documented $(2,13)$. Cuta- neous anthrax, acquired from the handling of infected animals or their products, is historically the most common. Infection occurs through a break in the skin that allows entry of the vegetative spores. Painless papules develop and become vesicular, then necrotic, often accompanied by significant edema, consistent with the expression of the edema factor and the lethal factor at the site of infection. The histology of cutaneous anthrax shows necrosis, lymphocytic infiltration, and edema; in contrast, the pathology associated with suppurative infections is characterized by infiltration of polymorphonuclear leukocytes, as is typical of a staphylococcal skin infection. Most cases of cutaneous anthrax resolve spontaneously, although antibiotic treatment is advised.

Inhalational anthrax occurs when spores are inhaled and is more often fatal. The initial clinical symptoms are not distinctive: fever, cough, and malaise. This progresses to significant dyspnea and cyanosis, but without pulmonary infiltrates in most cases. Instead, anthrax patients characteristically have mediastinitis (observed on chest films as a widened mediastinum) 
and marked pleural effusions. This progresses to what was considered to be septic shock, coma, and death. The pathophysiology of inhalational anthrax in humans has been assumed to be similar to shock associated with endotoxin (or LPS): an outpouring of proinflammatory cytokines, notably TNF- $\alpha$ and IL-1. This was based on the animal data suggesting that the lysis of infected macrophages would release large amounts of cytokines into the circulation. However, as a Gram-positive organism, B. anthracis lacks LPS, and there are few data to document that components of the organism potently trigger cytokine release. Nor has it been established that anthraxinfected macrophages in vivo release such large amounts of cytokines as to cause the sepsis syndrome. This distinction is clinically important, as therapies being introduced to treat septic shock that target TNF- $\alpha$ and other cytokines might not be appropriate for anthrax-associated shock due to an entirely different pathophysiology.

Macrophages have been found to be important in the pathophysiology of anthrax (14). Murine models of infection demonstrated rapid lysis of macrophages, and presumably release of intracellular cytokines, as an important consequence of anthrax toxin activity. Macrophage-depletion studies also implicated these cells as the target for the activity of toxin. A major distinction was made between strains of mice with susceptible macrophages, such as the $\mathrm{BALB} / \mathrm{c}$ and the C57BL "resistant" strains of mice, with the implication that resistant macrophages would not undergo lysis and release cytokines. However, a direct demonstration of how lethal toxin actually kills either of these types of mice, and whether macrophage susceptibility is involved, was lacking.

In the experiments detailed by Moayeri et al. (7), the effects of lethal toxin on $\mathrm{BALB} / \mathrm{c}$ and $\mathrm{C} 57 \mathrm{BL} / 6 \mathrm{~J}$ mice were meticulously compared. Although the timing of the pathological events in the two genetic backgrounds was somewhat different, the end result was the same: death characterized by tissue hypoxia, extensive liver necrosis, and pleural edema. There was no evidence of TNF- $\alpha$ or cytokine production in either strain of mice. The pathological features of endotoxin-mediated shock were not observed. The most prominent pathology associated with anthrax lethal toxin was extensive tissue necrosis and hypoxic damage in the liver, spleen, and bone marrow. These central effects of tissue hypoxia were documented by elevated levels of erythropoietin in the mice. There was no vascular collapse associated with shock, and no evidence of a consumption coagulopathy, nor were there endothelial changes typical of intravascular coagulation or renal dysfunction, the expected consequences of a process analogous to endotoxin-mediated shock. As the C57BL mice have macrophages that are "sensitive" to anthrax lethal toxin, in contrast to the $\mathrm{BALB} / \mathrm{c}$ mice, these results demonstrate that macrophage susceptibility to toxin is not critical in the host response. The general lack of a cytokine response to lethal toxin is consistent with toxin-mediated MAPK cleavage in vitro, as MAPKs are important in cytokine signaling in response to infection. The pathology observed in the mice correlates reasonably well with published studies of human anthrax. The prominence of cyanosis and hypoxia, in the absence of pulmonary infiltration, may be explained by central effects of the toxin that cause hypoxic injury in many tissues.

This analysis of the pathological effects of the $B$. anthracis lethal toxin (7) should help focus future studies of optimal therapy for patients exposed to this organism. These results make clear that anthrax patients exhibit a unique pathophysiology and should not be considered to have generic shock analogous to Gram-negative sepsis. Exactly how the lethal factor produces such profound tissue hypox- ia, what metabolic processes are affected in the liver and elsewhere, and how these effects may be blocked will require further studies. It should also be noted that despite the long interest in this pathogen, as well as the availability of classical genetic data, sophisticated molecular genetic tools, and 2-Å modeling information, the work of Moayeri et al. (7) relied predominantly on meticulous observation and the careful documentation of animal pathology, techniques that have been used successfully to study infectious diseases for the last century.

1. Dirckx, J.H. 1981. Virgil on anthrax. Am. J. Der matopathol. 3:191-195.

2. Shafazand, S., Doyle, R., Ruoss, S., Weinacker, A., and Raffin, T.A. 1999. Inhalational anthrax: epidemiology, diagnosis, and management. Chest. 116:1369-1376

3. Spencer, R.C. 2003. Bacillus anthracis. J. Clin Pathol. 56:182-187.

4. Mock, M., and Fouet, A. 2001. Anthrax. Annu. Rev. Microbiol. 55:647-671.

5. Petosa, C., Collier, R.J., Klimpel, K.R., Leppla, S.H., and Liddington, R.C. 1997. Crystal structure of the anthrax toxin protective antigen. Nature. 385:833-838.

6. Bradley, K.A., Mogridge, J., Mourez, M., Collier, R.J., and Young, J.A. 2001. Identification of the cellular receptor for anthrax toxin. Nature. 414:225-229.

7. Moayeri, M., Haines, D., Young, H.A., and Leppla, S.H. 2003. Bacillus anthracis lethal toxin induces TNF- $\alpha$-independent hypoxia-mediated toxicity in mice. J. Clin. Invest. 112:670-682. doi:10.1172/JCI200317991.

8. Cunningham, K., Lacy, D.B., Mogridge, J., and Collier, R.J. 2002. Mapping the lethal factor and edema factor binding sites on oligomeric anthrax protective antigen. Proc. Natl. Acad. Sci. U. S. A 99:7049-7053.

9. Leppla, S.H. 1982. Anthrax toxin edema factor: a bacterial adenylate cyclase that increases cyclic AMP concentrations of eukaryotic cells. Proc. Natl. Acad. Sci. U. S. A. 79:3162-3166.

10. Pellizzari, R., Guidi-Rontani, C., Vitale, G., Mock, M., and Montecucco, C. 1999. Anthrax lethal factor cleaves MKK3 in macrophages and inhibits the LPS/IFNgamma-induced release of NO and TNFalpha. FEBS Lett. 462:199-204.

11. Milne, J.C., Furlong, D., Hanna, P.C., Wall, J.S., and Collier, R.J. 1994. Anthrax protective antigen forms oligomers during intoxication of mammalian cells. J. Biol. Chem. 269:20607-20612.

12. Abrami, L., Liu, S., Cosson, P., Leppla, S.H., and van der Goot, F.G. 2003. Anthrax toxin triggers endocytosis of its receptor via a lipid raft-mediated clathrin-dependent process. J. Cell Biol. 160:321-328.

13. Dixon, T.C., Meselson, M., Guillemin, J., and Hanna, P.C. 1999. Anthrax. N. Engl. J. Med. 341:815-826.

14. Hanna, P.C., Acosta, D., and Collier, R.J. 1993. On the role of macrophages in anthrax. Proc. Natl. Acad. Sci. U. S. A. 90:10198-10201. 